
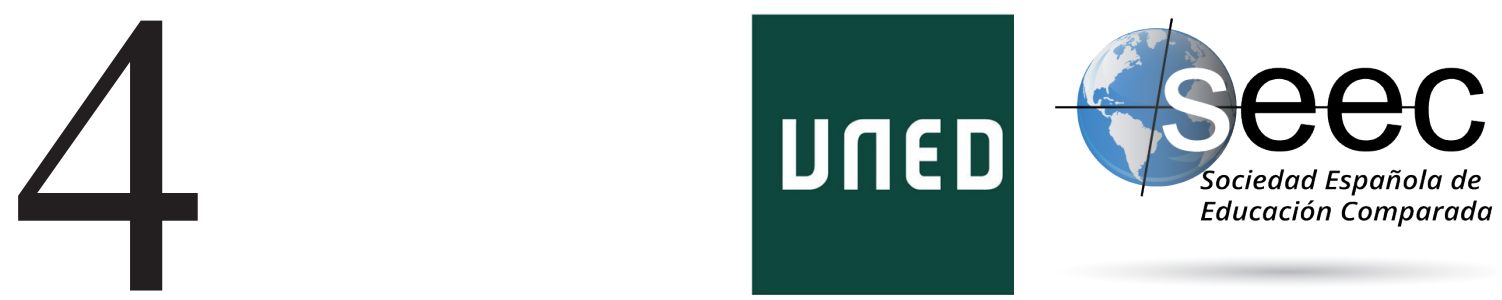

\title{
El inglés en educación obligatoria. Una mirada comparada a los casos de España y Holanda
}

\author{
English in compulsory education. A comparative \\ view of the cases of Spain and the Netherlands
}

\section{María González Villarón*}

DOI: $10.5944 /$ reec.30.2017.18694

\section{Recibido: 22 de marzo de 2017 Aceptado: 9 de mayo de 2017}

\footnotetext{
* María González Villarón: Doctora en Ciencias de la Educación por la Universidad Complutense de Madrid. Premio Nacional de Tesis Doctorales en Educación Comparada «Pedro Rosselló». Licenciada en Psicopedagogía y Maestra en Educación Infantil. Actualmente trabaja como maestra especialista impartiendo enseñanza bilingüe en inglés en el CEIP Cantos Altos de la Comunidad de Madrid. Ha participado en diferentes programas de formación en distintos países del extranjero. Su actividad investigadora se centra en la enseñanza de las lenguas extranjeras y en su desarrollo dentro de los distintos sistemas educativos. Datos de contacto: E-mail: mgvillaron@hotmail.com
} 


\title{
Resumen
}

El aprendizaje de lenguas extranjeras se ha convertido actualmente en uno de los objetivos que mayor interés despierta en los sistemas educativos. Por esta razón, revisar los procedimientos que se ponen en marcha para mejorar su calidad y eficiencia resulta una cuestión realmente necesaria. Hacerlo, además, desde una mirada comparada, aludiendo a uno de los países que posee un mayor dominio en el manejo de esta lengua, es un camino apenas trabajado. El objetivo de este artículo es contribuir a un mejor conocimiento y comprensión de las políticas e iniciativas llevadas a cabo en el ámbito de la enseñanza del inglés en España y Holanda con el fin de sugerir posibles aspectos susceptibles de mejora para nuestro sistema educativo. Para ello, en primer lugar, se presenta la descripción de los aspectos más significativos de la estructura y el funcionamiento de la enseñanza del inglés en cada una de las etapas que componen la enseñanza obligatoria de los dos países. Desde este marco, se realiza, posteriormente, el análisis comparado. Finalmente, se exponen las conclusiones halladas y se proponen, a la luz de los resultados, algunas líneas de reflexión que podrían contribuir a la mejora de la enseñanza y el aprendizaje de las lenguas extranjeras en la etapa de educación obligatoria en nuestro país.

Palabras clave: educación bilingüe; enseñanza y aprendizaje de una segunda lengua; inglés; España; Holanda

\begin{abstract}
The learning of foreign languages has now become one of the objectives that arouses most interest in the educational systems. For this reason, reviewing the procedures implemented to improve its quality and efficiency is a really necessary issue. Doing so, moreover, from a comparative perspective, alluding to one of the countries that has a high ability in the use of this language, is a road which has hardly been developed. The aim of this article is to contribute to a better knowledge and understanding of the policies and initiatives carried out in the field of English teaching in Spain and the Netherlands in order to suggest possible aspects that may improve our educational system. To do this, firstly, the description of the most significant aspects of the structure and functioning of the teaching of English of the two countries is presented, detailing each of the stages of compulsory education. Then, within this framework, a comparative analysis is performed. Finally, the conclusions found are presented, and based on these results, some lines of reflection are proposed that could contribute to the improvement of the teaching and learning of the foreign languages at compulsory education stage in our country.
\end{abstract}

Key Words: Bilingual Education; Second Language Instruction and Learning; English; Spain; The Netherlands 


\section{Introducción}

La enseñanza de lenguas extranjeras constituye hoy en día uno de los principales retos a los que los sistemas educativos deben hacer frente. La preocupación por desarrollar modos de enseñanza eficientes en este terreno suscita gran interés en gobiernos y administraciones y es, sin duda, uno de los objetivos mejor asentados en los diferentes programas educativos europeos. Una inquietud que, de igual modo, se encuentra respaldada por el compromiso adquirido con las distintas políticas europeas encaminadas a fomentar el multilingüismo. De tal forma que la mejora de la calidad y la eficiencia del aprendizaje de idiomas se sitúa hoy en día dentro de los objetivos clave del marco estratégico Educación y formación 2020 (UNIÓN EUROPEA, 2009).

Desde hace algunos años en nuestro país se vienen realizando importantes esfuerzos en este campo con el ánimo de mejorar una situación que, si bien, a la luz de los datos, no invita al optimismo, sí reconoce una relativa mejoría a lo largo de los últimos tiempos. Las escasas evidencias a nivel nacional sobre la competencia lingüística en inglés en primaria y secundaria han sido más que suficientes para apuntar que, en efecto, los alumnos españoles no contaban con un alto rendimiento en esta materia. Según La evaluación de la enseñanza y aprendizaje de la lengua inglesa (MECD, 2002a; MECD, 2004), primer y único análisis nacional realizado por el entonces Instituto Nacional de Calidad y Evaluación, la mayor parte de los estudiantes se situaba en los niveles medios establecidos para esta prueba. Por otro lado, un escaso $3 \%$ alcanzaba los niveles más altos en la etapa de secundaria. El estudio ponía de manifiesto, asimismo, grandes dificultades en las áreas de expresión oral y escrita. A la vista de estos datos y en coherencia con las premisas europeas, las administraciones educativas no dudaron de la necesidad de poner en marcha diversas medidas. Acciones que pasaban no solo por adelantar el comienzo de la edad de aprendizaje de las lenguas extranjeras, sino también y, sobre todo, por la implantación de programas bilingües. Si bien, esta última iniciativa no era realmente novedosa. En 1996 el Ministerio de Educación y el British Council firmaron un convenio en el que por primera vez se promovía una enseñanza bilingüe en centros públicos a partir de un currículo integrado: su implantación progresiva fue abriendo camino a esta modalidad de enseñanza. Por su parte el Ministerio de Educación, además de este acuerdo, hacía pública en 2010 la primera política global en este terreno: el Programa Integral de Aprendizaje de las Lenguas Extranjeras 2010-20 (PIALE) (MECD, 2011a). Un ambicioso plan cuyo principal objetivo era incrementar, en un plazo no superior a 10 años, el conocimiento de lenguas extranjeras en España. Lamentablemente solo se publicó la primera fase.

Comenzaba así una importante andadura cuyo fin no es otro que el de mejorar la competencia lingüística de la población española. Un objetivo que parece ir por el buen camino, puesto que los informes europeos sitúan a nuestro país como un ejemplo de promoción temprana de una segunda lengua (EURYDICE, 2008a). Investigadores de renombre, como Do Coyle, señalan que España se está convirtiendo en uno de los países más experimentados en la implantación de programas de inmersión lingüística (LASAGABASTER y RUIZ DE ZAROBE, 2010). A este fenómeno también se ha referido el profesor Baetens-Beardsmore, reconocida figura en el campo de la educación bilingüe, como el «tsunami español en el Aprendizaje Integrado de Contenidos y Lenguas» (MECD, 2011b). 
Ahora bien ¿̇son adecuadas las medidas que se están llevando a cabo en España? ¿Están correctamente dirigidas? ¿Distan mucho de las puestas en marcha por aquellos países que demuestran un mejor dominio de las lenguas extranjeras? El objetivo de este artículo es analizar desde una mirada comparada estas cuestiones, fijando la atención en uno de los países cuya habilidad en el dominio y manejo de las lenguas extranjeras es de sobra conocida y corroborada, como es Holanda.

Estas páginas, por tanto, presentan un sucinto recorrido por las medidas implantadas en la enseñanza de lenguas extrajeras en España y Holanda. El fin principal no es otro que el de contribuir a un mejor conocimiento de la situación del aprendizaje de idiomas y sugerir, a la vez, posibles aspectos susceptibles de mejora para nuestro sistema educativo.

\section{Aspectos metodológicos}

El artículo, como ya se ha adelantado, se propone comparar la estructura y el funcionamiento de la enseñanza de la lengua inglesa, lengua extranjera mayoritariamente hablada en ambos territorios. De esta manera, la selección de los sistemas educativos ha respondido principalmente a la capacidad de los alumnos para utilizar dicha lengua. Para ello se han tenido en cuenta los resultados que presenta el Estudio Europeo de la Competencia Lingüística (EECL) (EUROPEAN COMMISSIÓN, 2012) en su primera y, hasta la fecha, única edición. Un estudio comparativo que proporciona datos sobre el dominio de las lenguas extranjeras de los alumnos europeos al término de la Educación Secundaria Obligatoria.

Estos datos sitúan a Holanda, tras Suecia y Malta, entre los tres mejores países de la Unión Europea en cuanto a la habilidad en lengua inglesa se refiere. El estudio pone de manifiesto, asimismo, importantes diferencias entre ambos países (Tabla 1).

Tabla 1. Distribución por niveles del Marco Común Europeo de Referencia para las

Lenguas (Marco de Referencia) (INSTITUTO CERVANTES, 2002) en las destrezas analizadas en el EECL para España y Holanda (EUROPEAN COMMISSIÓN, 2012: 42-43).

\begin{tabular}{|c|c|c|c|c|c|}
\hline & PRE-A1 & A1 & A2 & $\overline{\text { B1 }}$ & $\overline{\text { B2 }}$ \\
\hline \multicolumn{6}{|c|}{ Comprensión oral } \\
\hline España & $31 \%$ & $32 \%$ & $13 \%$ & $12 \%$ & $12 \%$ \\
\hline Holanda & $3 \%$ & $10 \%$ & $10 \%$ & $18 \%$ & $59 \%$ \\
\hline \multicolumn{6}{|c|}{ Comprensión escrita } \\
\hline España & $18 \%$ & $40 \%$ & $12 \%$ & $12 \%$ & $18 \%$ \\
\hline Holanda & $4 \%$ & $21 \%$ & $15 \%$ & $22 \%$ & $38 \%$ \\
\hline \multicolumn{6}{|c|}{ Expresión escrita } \\
\hline España & $14 \%$ & $31 \%$ & $26 \%$ & $20 \%$ & $9 \%$ \\
\hline Holanda & $1 \%$ & $9 \%$ & $30 \%$ & $48 \%$ & $12 \%$ \\
\hline
\end{tabular}

Por otra parte, la selección de las unidades de análisis no solo ha respondido a este criterio, sino que obedece también a las grandes divergencias existentes en cuanto al modelo educativo. Los datos arrojados por los últimos estudios de la OCDE (2012), así como por la red Eurydice (2008b), señalan que Holanda es uno de los países con mayor 
tradición en cuanto a la autonomía escolar. Esto contrasta con los rasgos de funcionamiento del modelo español, que presenta una tradición de autonomía escolar mucho más centralizada (Bolívar, 2010). Sin ir más lejos, el citado informe de la OCDE recoge que el $86 \%$ de las decisiones que conciernen a la educación en Holanda se toman en los centros frente al $24 \%$ en el caso español. Holanda aventaja en este aspecto a todos los países de la OCDE (OECD, 2012).

De esta forma, a pesar de las diferencias existentes en cuanto a extensión geográfica y situación de sus lenguas de origen, el estudio de estos dos países puede resultar de gran interés.

Para realizar este análisis se ha utilizado una metodología comparativa, siguiendo las fases propuestas por García Garrido (1996), bajo las cuales se estructura este artículo. En primer lugar, se ha llevado a cabo el estudio descriptivo (fase analítica). El análisis de este contenido se ha realizado a través de la consulta de fuentes primarias, secundarias y auxiliares (BEREDAY, 1968). Esta información se ha completado con la observación directa en centros escolares y entrevistas a expertos. En segundo lugar, se ha abordado el estudio comparativo (fase sintética) en el que se incluye la yuxtaposición de los datos atendiendo a las categorías de análisis y las conclusiones comparativas. Si bien, en este caso estas últimas aparecen en un epígrafe aparte junto a una serie de propuestas, realizadas a la luz de estas reflexiones, que podrían contribuir a la mejora de la situación española.

\section{Descripción de la estructura y funcionamiento de la enseñanza del inglés en la educación obligatoria}

\subsection{Holanda}

\subsubsection{Educación primaria}

Los alumnos holandeses comienzan a aprender inglés relativamente tarde. La lengua inglesa es una asignatura obligatoria a partir de los 10 años y durante esta etapa es un área con poco peso curricular. Si bien, la carga lectiva no está regulada a nivel legal, generalmente se sitúa entre los 30 y los 60 minutos semanales (THIJS, TRIMBOS, TUIN, BODDE, DE GRAAFF, 2011; ROSE, 2016), algo que resulta realmente asombroso si se compara con el resto de los países europeos (EURYDICE, 2012). Su docencia recae sobre el maestro tutor.

Su currículo, escasamente prescriptivo, presenta cuatro objetivos específicos que se enmarcan dentro de un enfoque comunicativo. Estos objetivos incurren en el trabajo de tres de las cuatro destrezas que componen el aprendizaje de una lengua extranjera (comprensión oral y escrita y expresión oral), relegando el desarrollo de la expresión escrita para la siguiente etapa (MINISTERIE VAN ONDERWIJS, CULTUUR EN WETENSCHAP, 2006a).

No obstante lo anterior, para reforzar su aprendizaje, en los últimos tiempos se está llevando a cabo la implantación progresiva de distintas experiencias de aprendizaje temprano de inglés que se engloban dentro del programa La internacionalización de la escuela primaria. Esta tendencia apunta hacia una doble dirección: reforzar y ampliar el tiempo lectivo y desarrollar programas de inmersión. Así pues, por un lado, desde 2010, se están implantando programas de aprendizaje temprano de lenguas extranjeras 
$\left(V V T O^{1}\right)$. En ellos la lengua inglesa se aprende o bien desde el grupo 1 (4 años), generalmente durante una hora a la semana, o bien desde el grupo 5 ( 8 años), en este caso la carga lectiva varía de una a tres horas semanales. Aunque no existe un currículo diferente, el Instituto de Desarrollo Curricular (SLO) publica una guía práctica con la concreción de los objetivos del área en distintos contenidos y actividades, organizados a lo largo de los cursos (BODDE, SCHIPPERS y KLEIN, 2008). La Organización holandesa para la internacionalización de la educación (Nuffic) ${ }^{2}$ completa estas directrices a través de la publicación de un manual de calidad (EUROPEES PLATFORM, 2011) que guía la práctica docente. En él se describen algunos aspectos metodológicos y diferentes pautas de evaluación tanto del programa como del alumnado. Respecto a este último punto, se estima que el alumnado al término de la etapa adquirirá, al menos, un nivel A2 (Marco de Referencia) en todas las destrezas trabajadas. Entre las primeras, asimismo, se describe el perfil pedagógico del docente, en la mayoría de los casos profesor generalista. Esta descripción hace referencia no solo a las capacidades lingüísticas, entre las que se espera que esté en posesión de un nivel B2 (Marco de Referencia), sino, sobre todo, a las habilidades didácticas y pedagógicas. Para garantizar la cohesión en el aprendizaje, las escuelas cuentan con la figura del coordinador del programa y pueden, además, optar al certificado de calidad de Escuela VVTO, lo que supone que el centro cumple con los requisitos nacionales estipulados.

Por otro lado, desde el curso 2014-15 bajo la coordinación de la Nuffic, se está implantando un proyecto piloto de educación bilingüe (TPO $\left.{ }^{3}\right)$ de cinco años de duración, cuyo fin es diseñar un programa bilingüe de alta calidad. Los alumnos de estos centros reciben entre el $30 \%$ y el $50 \%$ de su horario en inglés, siendo el currículo holandés la principal referencia. El programa cuenta con una fuerte orientación internacional. La evaluación de estos proyectos corre a cargo de la Inspección Educativa y la formación de profesorado queda en manos de las escuelas.

Sin embargo, estas acciones no son ajenas a la polémica que enfrenta a quienes ven en la enseñanza de lenguas una clara ventaja para el desarrollo del alumnado y a aquellos que defienden que estos primeros años deben emplearse en el afianzamiento del neerlandés, lengua oficial del país. Estos últimos amparados bajo la actual Ley de Educación Primaria (WET VAN 2 JULI 1981, OP HET BASIS ONDERWIJS), que establece la lengua neerlandesa como la única lengua de instrucción.

\subsubsection{Educación secundaria}

Es en esta etapa donde Holanda cuenta con una sólida y cuidada política educativa de aprendizaje de lenguas extranjeras que contempla, por un lado, una amplia oferta de idiomas, entre los que se encuentra incluso el chino y, por otro, una dilatada experiencia en la enseñanza de inglés. No obstante, a pesar de las citadas ventajas, la preocupación por mejorar la competencia lingüística, sobre todo en inglés, sigue siendo una línea prioritaria de actuación.

La lengua inglesa es una asignatura obligatoria impartida por profesores especialistas a lo largo de toda la etapa. En el ciclo inferior, desde 2014 y junto con neerlandés y

\footnotetext{
$1 \quad$ Vroeg Vremdetalenonderwijs

2 La Organización holandesa para la internacionalización de la educación (Nuffic) ha sido conocida hasta hace relativamente poco como la Plataforma Europea. Cabe advertir que la mayoría de los documentos a los que se hace referencia en este artículo están publicados por la entonces Plataforma Europea.

Tweetalig Onderwijs
} 
matemáticas, es, además, una asignatura instrumental. Este carácter instrumental se ha traducido en la obligatoriedad de realizar pruebas de diagnóstico y en la formulación de unos objetivos intermedios más específicos (SLO, 2017) pero, no ha afectado a la organización del tiempo que sigue recayendo, en gran parte, en manos de los centros. Para esta etapa la ley únicamente establece que dos tercios del total del tiempo escolar se dediquen a la consecución de los objetivos generales del primer ciclo, otorgándole autonomía a la escuela para decidir sobre la organización del tiempo restante (WET VAN 29 JANUARI 1993, OP HET VOORTGEZET ONDERWIJS). Los datos que presenta el Instituto de Estadística Educativa sitúan el tiempo de enseñanza obligatorio de esta asignatura en torno a las dos horas y media semanales. Estos datos señalan, además de eso, que el inglés es, por debajo de matemáticas y junto a neerlandés, una de las asignaturas que se imparten con mayor frecuencia en el tiempo de libre asignación (FONTEIN, PRÜFER, DE VOS y VLOET, 2016). En el ciclo superior la carga lectiva está estipulada para el total de la etapa. Su organización varía en función de los centros y de los itinerarios, no obstante, una estimación orientativa permite situarla en torno a las tres horas y media semanales (FASOGLIO, DE JONG, PENNEWAARD, TRIMBOS y TUIN, 2015).

El currículo recoge para el ciclo inferior ocho objetivos que hacen referencia a las cuatro destrezas, aunque le confieren una mayor importancia a la adquisición de una buena comprensión oral y de un vocabulario básico, limitando el desarrollo de la expresión escrita a unos mínimos funcionales (MINISTERIE VAN ONDERWIJS, CULTUUR EN WETENSCHAP, 2006b). Para guiar la práctica educativa el SLO ofrece una concreción de estos objetivos (TRIMBOS, 2007) a partir del enfoque por tareas (ELLIS, 2003) y unos objetivos específicos diferenciados por itinerarios para cada una de las destrezas, siguiendo las directrices del Marco de Referencia (SLO, 2017). En el ciclo superior los objetivos se diferencian por itinerarios y están definidos en función de las destrezas, añadiendo el área de literatura en los itinerarios no profesionales y el de aspectos culturales para los itinerarios profesionales.

Junto a estas indicaciones el SLO ha establecido, sobre la base del Marco de Referencia, los niveles orientativos que debe obtener el alumnado al finalizar la etapa de educación secundaria según las distintas opciones educativas: A2 para formación profesional, B1 para el itinerario general y B2 para el más académico (ERK, 2017).

La lengua inglesa debe ser evaluada en el examen final nacional holandés. En esta prueba únicamente es obligatorio conocer el nivel de comprensión escrita; sin embargo, generalmente se valora el nivel de adquisición de las cuatro destrezas y el área de literatura o de aspectos socioculturales, según corresponda. Del diseño y el desarrollo de la evaluación de la comprensión escrita se encarga el Instituto Central para la Medición y el Desarrollo (Cito ${ }^{4}$. La evaluación de las otras destrezas recae en manos de los centros, aunque el SLO, tal y como lo refiere Daniela Fasoglio, coordinadora del área de lenguas extranjeras en educación secundaria del SLO: «está tratando de crear una red que permita guiar y supervisar estas pruebas» (Entrevista, 4 de julio de 2013). Asimismo, entre las nuevas políticas educativas el gobierno está diseñando un examen nacional de inglés que se pondrá en marcha durante el curso 17-18 para el alumnado de las ramas más básicas de formación profesional, puesto que hasta ahora este alumnado estaba exento de su realización.

$4 \quad$ Het Centraal Instituut voor Toetsontwikkeling 
La educación secundaria holandesa cuenta, por otro lado, con una gran trayectoria en la implantación de programas bilingües $\left(\mathrm{TTO}^{5}\right)$ en los itinerarios más académicos de la educación secundaria. La educación bilingüe comenzó, en efecto, en 1989 a partir del interés y esfuerzo de un grupo de padres y profesores altamente motivados. De ahí deriva el carácter eminentemente práctico, forjado a través de los años, de un proceso que se ha convertido en un programa educativo completo a partir de una experiencia a pequeña escala (ADMIRAAL, DE BOT y WESTHOFF, 2006). Una experiencia considerada por muchos «una de las innovaciones más exitosas en la educación holandesa de las últimas dos décadas» (MALJERS, 2007, p.131).

En estos programas los alumnos no solo estudian otra lengua, sino que participan en un programa intercultural orientado hacia una dimensión internacional (NUFFIC, 2017). El tiempo lectivo impartido en inglés ocupa un 50 \% del horario en el nivel inferior; sin embargo, en el nivel superior se reduce a un 30 \% y «la razón es muy simple: el examen nacional es en neerlandés» (Daniela Fasoglio. Entrevista, 4 de julio de 2013). No hay un modelo bilingüe único prescrito, aunque sí se recogen unas notas legales diseñadas por la Nuffic que hacen referencia a las áreas impartidas en lengua extranjera, horas de exposición a la lengua, grado de desarrollo del currículo holandés y requisitos para la incorporación de alumnos al proyecto (EUROPEAN PLATFORM, 2012).

Existe un perfil concreto de profesorado que contempla una doble vertiente, por un lado la destreza lingüística, para la que se exige un nivel B2 (Marco de Referencia), y por otro, la formación pedagógica y didáctica. Al finalizar el primer ciclo se espera que el alumnado haya adquirido un nivel de competencia B2 (Marco de Referencia) y al finalizar la etapa el nivel A2 en lenguas establecido por la Organización de Bachillerato Internacional. La red de escuelas bilingües coordinada por la Nuffic cuenta con un proceso de evaluación continua que incluye visitas a los centros por parte de expertos encargados de valorar si cumplen los estándares establecidos y de expedir los títulos de Escuela Bilingüe. No obstante, es la Inspección Educativa, en último término, la responsable del seguimiento y de la evaluación de la calidad de estos proyectos.

En los itinerarios no profesionales, los centros pueden optar por un programa de refuerzo de las lenguas extranjeras $\left(V T O^{6}\right)$. En este caso se aumenta el tiempo dedicado a la enseñanza de inglés media, una o dos horas a la semana, siempre fuera del horario lectivo o dentro de unas horas estipuladas para el estudio personal. Al finalizar la etapa los alumnos obtienen un diploma de Cambridge que certifica el nivel alcanzado.

\subsection{España}

\subsubsection{Educación primaria}

En nuestro país el aprendizaje de una segunda lengua se introduce en el segundo ciclo de educación infantil (LOE, art.14.5) y tiene continuidad a lo largo de toda la escolaridad obligatoria. Dependiendo de cada administración educativa, supone en esta etapa una carga lectiva de entre dos y cuatro horas semanales, impartidas siempre por un maestro especialista.

Adquirir «una competencia comunicativa básica que les permita expresar y comprender mensajes sencillos y desenvolverse en situaciones cotidianas» (LOMCE, art.7) es la finalidad última que se persigue a través de su enseñanza. Para ello, el currículo describe de forma pormenorizada los contenidos, los criterios de evaluación y los estándares de

$5 \quad$ Tweetalig Onderwijs

$6 \quad$ Versterkt taalonderwijs o Fast Lane English 
aprendizaje (RD 126/ 2014, ANEXO I) para esta asignatura. Incide también en el desarrollo de las cuatro destrezas, descritas tal y como aparecen en el Marco de Referencia.

Si bien, con el objetivo de fortalecer la enseñanza de idiomas, las Comunidades Autónomas han puesto en marcha programas de refuerzo del aprendizaje de lenguas que, en los últimos tiempos, están dando paso a distintos planes de inmersión lingüística o proyectos bilingües. El desarrollo del convenio antes mencionado entre el Ministerio de Educación y el British Council en 1996 sirvió de base para el diseño de estos programas. Los cuales al amparo de la LOE (RD 1513/2006, DISPOSICIÓN ADICIONAL SEGUNDA), hicieron efectiva la posibilidad de impartir parte de las áreas de aprendizaje en lengua extranjera. En este caso son las administraciones educativas las encargadas de establecer la normativa para su región, de tal forma que la gran autonomía con la que cuentan y la escasa regulación emanada desde el gobierno central dificulta una aproximación específica. A esto se suma su reciente implantación, puesto que los programas más antiguos cuentan con poco más de una década de experiencia. A grandes rasgos, pueden extraerse algunas de las características, compartidas en las dos etapas, que contribuyan a clarificar someramente el estado de la cuestión. En todos ellos, al menos un área o materia no lingüística se imparte en inglés. La exposición a la lengua extranjera se sitúa entre un $20 \%$ y un 50 \% del horario, si bien, muchas administraciones conceden autonomía a los centros para ampliar este horario, siempre que no vaya en detrimento del desarrollo de otras áreas curriculares. En ningún caso, excepto en la Comunidad Autónoma de Madrid (ORDEN 5958/2010), existe un currículo específico para el área de inglés. El profesorado encargado de impartir las materias no lingüísticas ha de poseer una habilitación para el desempeño de la docencia bilingüe que acredita el conocimiento de la lengua. La mayor parte de las Comunidades Autónomas exigen un nivel B2 (Marco de Referencia), asimismo, todas cuentan con la figura del coordinador bilingüe y con apoyo en el aula de auxiliares de conversación nativos. En cuanto a la evaluación, en los casos que se especifica, el alumnado debe alcanzar, al menos, el nivel B1 al finalizar la Educación Secundaria Obligatoria.

\subsubsection{Educación secundaria}

Durante esta etapa el inglés es una asignatura obligatoria, impartida por profesores especialistas. El tiempo de enseñanza está estipulado por las administraciones, situándose entre las tres y las cuatro horas semanales.

$\mathrm{Al}$ igual que ocurre en primaria el currículo describe detalladamente, sobre la base del Marco de Referencia, contenidos, criterios de evaluación y estándares de aprendizaje que trabajan equitativamente sobre las cuatro destrezas de aprendizaje (RD 1105/2014, ANEXO I). La evaluación es, por tanto, un proceso continuo, global y formativo que se realiza en función de los diferentes elementos del currículo a lo largo de todos los cursos.

No existe una orientación concreta sobre el nivel que ha de alcanzar el alumnado al finalizar la etapa, aunque lo cierto es que a efectos legales se sobreentiende que contará con un nivel B1.

Asimismo, España estrena durante este curso una evaluación nacional, requisito imprescindible para la obtención del título de Educación Secundaria Obligatoria (LOMCE, art.29) y de Bachillerato (LOMCE, art.36 bis). En ella se valoran, entre otros, los conocimientos de inglés al término de cada uno de los ciclos de esta etapa en cualquiera de las opciones educativas: aún se desconoce su diseño. Hasta la fecha la única 
evaluación existente era la Prueba de Acceso a la Universidad (PAU), un examen de entrada a la universidad realizado al finalizar la educación secundaria en el que la primera lengua extranjera formaba parte de las materias obligatorias a evaluar. Su diseño, bajo las directrices del Ministerio, recaía en manos de las administraciones, con lo cual se hace difícil definir una estructura común al territorio español. A pesar de ello, como rasgo general la mayoría de ellas evaluaban únicamente las destrezas de comprensión y expresión escrita. En algún caso aislado también se valoraban la comprensión oral y los conocimientos de fonética.

\section{Estudio comparado}

Tras el análisis descriptivo, la comparación permite reflejar posibles convergencias y divergencias entre los dos sistemas. Con el fin de facilitar su lectura, la yuxtaposición de los datos se presenta a partir de las categorías de comparación que permiten delimitar y clasificar los datos expuestos durante la fase analítica. Estas categorías son: edad de comienzo del aprendizaje, carga lectiva, currículo, profesorado y programas de refuerzo.

En cuanto a la edad de comienzo del aprendizaje de una segunda lengua extranjera, cabe advertir que los alumnos holandeses comienzan su andadura por el aprendizaje del inglés unos seis o siete años más tarde que los españoles. Las políticas educativas holandesas conceden un alto valor al aprendizaje y afianzamiento de neerlandés y consideran este un requisito imprescindible para lanzarse a manejar un segundo código lingüístico. Aun así, no son pocos aquellos que reconocen las ventajas de aprender un segundo idioma a edades tempranas. En medio de esta controversia, las cifras que se manejan hasta el momento revelan que más de la mitad de las escuelas de educación primaria de Holanda todavía no han adoptado ningún modelo de enseñanza temprana de lenguas (THIJS, TRIMBOS, TUIN, BODDE, DE GRAAFF, 2011).

Por otro lado, el alumnado holandés no solo comienza tarde a aprender la lengua inglesa, sino que lo hace durante escaso tiempo. En los primeros años la carga lectiva semanal de esta asignatura es, cuanto menos, 60 minutos superior en España. Incluso, el incremento de estas horas de aprendizaje a partir de la implantación de programas de aprendizaje temprano de lenguas extranjeras en Holanda no llega ni tan si quiera a alcanzar aquel que recogen las disposiciones legales establecidas en España para esta asignatura. Por lo tanto, a pesar de los esfuerzos puestos en marcha, el inglés en Holanda mantiene «una posición humilde» (THIJS, TRIMBOS, TUIN, BODDE, DE GRAAFF, 2011: 16) dentro del currículo de educación primaria. Sin embargo, estas diferencias desaparecen por completo en educación secundaria. A este respecto, cabe destacar que la organización de la enseñanza del inglés en esta etapa superior en España es algo menos compleja, no solo por la menor autonomía con la que cuentan los centros sino, sobre todo, por su estructura. La amplia ramificación de este tramo educativo en Holanda confiere una mayor diversidad de escenarios educativos.

A estas particularidades se le debe añadir aquellas que se desprenden del currículo, entendiendo como trasfondo que gran parte de estas se encuentran intrínsecamente unidas a aquellas divergencias halladas en torno a la autonomía de centro. Así, pues, los dos países no difieren tanto en su objetivo general, bajo el cual subyace en ambos casos un enfoque comunicativo, si no, sobre todo, en la descripción de sus elementos. El currículo holandés, escasamente prescriptivo junto a la amplia autonomía de los centros redundan en un incremento, en número y en importancia, de las funciones de estos 
últimos respecto al diseño de la política curricular. Por el contrario, España cuenta con una mayor definición del marco curricular que reduce de manera significativa el grado de concreción curricular de los centros. En este sentido, el informe PISA 2012 pone de manifiesto que el 65,3 \% de la varianza de los resultados en los Países Bajos se atribuye a las diferencias entre centros, mientras que en España este porcentaje desciende al 17,1 \% (MECD, 2013).

Conviene destacar, asimismo, que ambos países ponen en marcha mecanismos de rendición de cuentas. En España estos mecanismos están definidos en términos de criterios de evaluación y estándares de aprendizaje evaluables para cada etapa, lo que le confiere una mayor estabilidad y sistematicidad. Sin embargo, nuestros vecinos holandeses cuentan con unos procedimientos de evaluación mucho más sólidos al término de la educación obligatoria.

En cuanto al profesorado, la diferencia principal viene marcada por las exigencias de formación en la etapa de educación primaria. En Holanda, durante estos primeros años, el profesorado encargado de impartir inglés es habitualmente un maestro generalista. En España, por el contrario, la docencia de esta asignatura recae siempre en manos de un maestro especialista.

Por otro lado, las distintas indicaciones que se realizan desde las nuevas políticas educativas dejan entrever en el caso holandés una exigencia formativa que apela, en gran medida, a los aspectos pedagógicos. En cambio, en España la mayoría de estas directrices hacen referencia no tanto a estas cuestiones sino al nivel de adquisición de la lengua inglesa.

Por último, en líneas generales, se puede decir que los programas de refuerzo de aprendizaje de las lenguas extranjeras comparten las directrices sobre las que se asientan; pero, un aspecto destacable es la importancia que Holanda concede en su diseño al desarrollo de lo que Van Ek (1993) se ha referido como «conciencia intercultural». Así pues, el foco de atención principal no reside tanto en mejorar la habilidad lingüística como en incrementar la orientación internacional. En cuanto a los programas bilingües, resulta difícil establecer una comparación entre ambos países, ya que, como se ha hecho referencia a lo largo de este artículo, no existe un modelo común al territorio español. Con la información ofrecida se puede decir que, a grandes rasgos, comparten características en cuanto al tiempo de exposición y las orientaciones metodológicas. Conviene, por lo tanto, en esta ocasión centrar el foco de atención mayoritariamente en los modos de proceder. La puesta en marcha de estos programas en el contexto holandés encierra importantes mecanismos de acción centrados en las escuelas y coordinados y supervisados por el gobierno. La evaluación constituye el pilar fundamental sobre el que se asienta su conducción y su difusión está sujeta a un periodo de prueba muy controlado. Durante este tiempo se dedican importantes esfuerzos al diseño de un marco común que guíe y garantice cierta uniformidad en las prácticas educativas, y que proporcione seguridad y apoyo a su profesorado. Este proceso contrasta con el modus operandi del gobierno y de las administraciones educativas españolas que, a pesar de su mayor experiencia en este terreno, organizan la implantación de estos programas con una lógica, en palabras de Bolívar (2010), más burocrática, que se aleja de potenciar el desarrollo institucional de los centros y los mecanismos de control de su calidad. 


\section{Conclusiones y propuestas de mejora para el caso español}

Como se puede observar tras el estudio comparativo, la preocupación por mejorar el aprendizaje de inglés está suscitando gran atención en las políticas educativas de ambos países. En términos generales, ambos sistemas comparten muchos de los elementos que configuran la enseñanza del inglés, si bien cada país, influido por diversos aspectos, ofrece un modo diferente de llevar a cabo esta práctica educativa.

En la base de estas divergencias se sitúa la cuestión de la autonomía de centros. Los diferentes modelos de gestión de los sistemas educativos convergen en distintos modelos de enseñanza de las lenguas extranjeras. No obstante, cabe advertir que en este terreno los nuevos modos de regulación de las políticas educativas acercan ligeramente las prácticas educativas. En el caso de Holanda las iniciativas adoptadas recientemente buscan conseguir una mayor uniformidad en el sistema escolar. En este sentido, la labor del SLO se ha hecho cada más necesaria a partir de una realidad que demanda indicaciones más precisas. Mientras tanto, en España la nueva ley educativa (LOMCE) parece dedicar parte de estos esfuerzos a reducir el control directo que se lleva a cabo por parte del gobierno y de las administraciones.

Desde el punto de vista de las iniciativas institucionales, España aventaja considerablemente a Holanda en el desarrollo de la enseñanza de las lenguas extranjeras en las etapas de educación infantil y primaria. Sin embargo, los Países Bajos cuentan con una gran experiencia en la promoción del aprendizaje de idiomas en las etapas superiores de la educación obligatoria. A pesar de estas diferencias, los modelos de enseñanza de las lenguas extranjeras parecen ir aproximándose, probablemente debido a la aplicación de las directrices y orientaciones emanadas de la Unión Europea y el Consejo de Europa. Al amparo de estas indicaciones, se considera importante continuar indagando en aquellas prácticas que pueden ayudar a mejorar los modelos de enseñanza de las lenguas extranjeras.

De este modo, tras este estudio, se pueden extraer algunas indicaciones que permiten reflexionar sobre la enseñanza del inglés en nuestro país. Así, pues, desde la riqueza que proporciona la mirada comparada se propone:

- Diseñar formas de gestión que deleguen parte de la toma de decisiones en las escuelas, aumentando su responsabilidad y el compromiso de colaboración. Para ello conviene reforzar el desarrollo institucional de los centros y diseñar un currículo menos prescriptivo y más flexible.

- Dotar de un mínimo de uniformidad las distintas iniciativas de refuerzo y aprendizaje de las lenguas que están surgiendo en las diferentes Comunidades Autónomas. En este sentido, sería adecuado diseñar un marco de actuación con unas directrices básicas que garantice el progreso hacia unos intereses comunes y sobre este, dotar de autonomía a las administraciones y a los centros. Además, procurar que estas iniciativas vengan precedidas por un programa definido de implantación que cuente con el desarrollo de experiencias piloto y que conceda mayor presencia a la orientación internacional.

- Asegurar que el diseño de los programas de aprendizaje temprano de lenguas extranjeras incida mucho más en cuestiones de índole didáctica y pedagógica. De este modo, sería importante que en este diseño se considere no solo la posibilidad de adelantar su aprendizaje o aumentar su carga lectiva, sino, sobre todo, las actividades a través de las que se enseña la lengua extranjera, procurando que estas estén siempre encaminadas a incrementar la calidad y la cantidad de interacciones lingüísticas. (JUAN, 2008) 
- Prestar mayor atención durante las primeras etapas al desarrollo de las habilidades de comprensión oral y escrita y de expresión oral, postergando ligeramente el desarrollo de la expresión escrita.

- Revisar los mecanismos de rendición de cuentas y trabajar en la elaboración y consolidación de unos indicadores nacionales que permitan obtener información sobre el grado de calidad de la enseñanza y el aprendizaje de lenguas extranjeras.

- Acompañar estas medidas con distintas políticas que incrementen y mejoren la exposición al inglés también fuera del entorno escolar (AUTOR, 2017).

\section{Referencias bibliográficas}

ADMIRAAL, W., DE BOT, K. and WESTHOFF, G. (2006): Evaluation of Bilingual Secondary Education in the Netherlands. Students' language proficiency, Educational Research and Evaluation, 12, 1, pp. 75 - 93.

BEREDAY, G. Z. F. (1968): El método comparativo en pedagogía (Barcelona, Herder).

BODDE, M., SCHIPPERS, S. en KLEIN, M. (2008): TULE Engels. Inhouden en activiteiten bij de kerndoelen (http://tule.slo.nl/Engels/F-KDEngels.html), consultado el 20 de diciembre de 2016.

BOLÍVAR, A. (2010): La autonomía de los centros educativos en España, Revista Participación Educativa, 13, pp.8-25.

ELLIS, R. (2003): Task-based language learning and teaching (Oxford, Oxford University Press).

ERK (2017): Europees Referntiekader Talen (http://www.erk.nl/leerling/), consultado el 23 de febrero de 2017.

EUROPEAN COMMISSION (2012): First European Survey on Language Competences: Final Report, Version 2.o. Education and Training (http://ec.europa.eu/ languages/policy/strategic-framework/documents/language-survey-finalreport_en.pdf), consultado el 29 de noviembre de 2016.

EUROPEAN PLATFORM (2012): Standard for bilingual education in English - havo/ vwo (https://www.nuffic.nl/en/publications/find-a-publication/standard-forbilingual-education.pdf), consultado el 10 de febrero de 2017.

EUROPEES PLATFORM (2011): Handleiding kwaliteit vvto Engels (http://www. europeesplatform.nl/vvto/wp-content/uploads/sites/7/2014/04/Handleidingkwaliteit-vvto-Engels.pdf), consultado el 3 de febrero de 2017.

EURYDICE (2008a): Key Data on Teaching Languages at School in Europe (Bruselas, European Commission).

EURYDICE (2008b): La autonomía escolar en Europa. Políticas y medidas (Madrid, MEC-CIDE).

EURYDICE (2012): Key Data on Teaching Languages at School in Europe 2012 (Bruselas, European Commission). 
FASOGLIO, D., DE JONG, K., PENNEWAARD, L., TRIMBOS, B. en TUIN, D. (2015): Moderne vreemde talen. Vakspecifieke trendanalyse 2015 (http://downloads. slo.nl/Repository/moderne-vreemde-talen-vakspecifieketrendanalyse-2015.pdf), consultado el 22 de enero de 2017.

FONTEIN, P., PRÜFER, P, DE VOS, K. en VLOET, A. (2016): IPTO: bevoegdheden en vakken in het vo. Eindrapport (http://www.voion.nl/downloads/5a16906c-136a4d1e-9258-04fce1617503), consultado el 8 de febrero de 2017.

GARCÍA GARRIDO, J. L. (1996): Fundamentos de Educación Comparada ( $3^{\mathrm{a}}$ ed.) (Madrid, Dykinson).

GONZÁLEZ VILLARÓN, M. y EGIDO GÁLVEZ, I. (2017): Factores explicativos del aprendizaje de la comprensión oral en lengua inglesa en educación secundaria: comparación entre España y Holanda, Revista Complutense de Educación, 28, 2 (http://dx.doi.org/10.5209/rev_RCED.2017.v28.n2.49634).

INSTITUTO CERVANTES (2002): Marco común europeo de referencia para las lenguas: aprendizaje, enseñanza y evaluación (Madrid, MEC).

JUAN, M. (2008): Contexto y contacto en el aprendizaje de lenguas extranjeras, IN. Revista Electrònica d'Investigació i Innovació Educativa i Socioeducativa, 1, o, pp.47-66 (http://www.in.uib.cat/pags/volumenes/vol1_numo/maria_garau/ index.html), consultado el 23 de abril de 2017.

LASAGABASTER, D. y RUIZ DE ZAROBE, Y. (2010): CLIL in Spain. Implementation, Results and Teacher Training (Cambridge, Cambridge Scholars Publishing).

LEY ORGÁNICA 2/2006 DE EDUCACIÓN (LOE), de 3 de mayo de 2006. En Boletín Oficial del Estado, 106, de 4 de mayo de 2006.

MALJERS, A. (2007): The Netherlands, en A. MALJERS, D. MARSH, AND D. WOLFF. Windows on CLIL: Content and Language Integrated Learning in the European Spotlight, pp.130-138 (La Haya, European Platform for Dutch Education, and Graz, European Centre for Modern Languages).

MECD (2002): Evaluación de la enseñanza y el aprendizaje de la lengua inglesa: Educación Primaria 1999. Informe final (Madrid, INCE).

MEC (2004): Evaluación de la enseñanza y el aprendizaje de la lengua inglesa. Educación Secundaria Obligatoria 2001. Informe final (Madrid, INECSE).

MECD (2011a): Programa Integral de Aprendizaje de Lenguas Extranjeras (https:// www.campuseducacion.com/files/programa-integral-aprendizaje-lenguasce-23-03-11.pdf), consultado el 28 de noviembre de 2016.

MECD (2011b): Revista Electrónica de didáctica del Español como Lengua Extranjera (RedELE), 22 (http://www.mecd.gob.es/dctm/redele/ Material-RedEle/Revista/2011_22/2011_redELE_22_ooentrevista. pdf?documentId=0901e72b8odbco07), consultado el 1 de diciembre de 2016. 
MECD (2013): PISA 2012. Programa Internacional para la Evaluación Internacional de los Alumnos. Informe español. Volumen I: resultados y contexto (Madrid, INEE).

MINISTERIE VAN ONDERWIJS, CULTUUR EN WETENSCHAP (2006a): De Kerndoelen Boekje Primair Onderwijs (La Haya, Ministerie van Onderwijs, Cultuur en Wetenschap).

MINISTERIE VAN ONDERWIJS, CULTUUR EN WETENSCHAP (2006b): Karakteriestieken en kerndoelen voor de onderbouw. Zwolle: Onderbouw VO (La Haya, Ministerie van Onderwijs, Cultuur en Wetenschap).

NUFFIC (2017): De Nederlandse organisatie voor internationalisering in onderwijs (https://www.nuffic.nl), consultado el 10 de febrero de 2017.

OECD (2012): Education at a Glance: OCDE indicators (https://www.oecd.org/edu/ EAG \%202012_e-book_EN_200912.pdf), consultado el 12 de enero de 2017.

ORDEN 5958/2010, de 7 de diciembre, de la Consejería de Educación, por la que se regulan los colegios públicos bilingües de la Comunidad de Madrid. En Boletín Oficial de la Comunidad de Madrid, 17, de 21 de enero de 2011.

REAL DECRETO 1513/2006, de 7 de diciembre, por el que se establecen las enseñanzas mínimas de la Educación Primaria. En Boletín Oficial del Estado, 293, de 8 de diciembre de 2006.

REAL DECRETO 126/ 2014, de 28 de febrero, por el que se establece el currículo básico de la Educación Primaria. En Boletín Oficial del Estado, 52, de 1 de marzo de 2014.

REAL DECRETO 1105/2014, de 26 de diciembre, por el que se establece el currículo básico de la Educación Secundaria Obligatoria y del Bachillerato. En Boletín Oficial del Estado, 3, de 3 de enero de 2015.

ROSE, P. (2016): Engels in het basisonderwijs (http://downloads.slo.nl/Repository/ engels-in-het-basisonderwijs-domeinbeschrijving.pdf), consultado el 30 de abril de 2017.

SLO (2017): Nationaal Expertisecentrum voor Leerplanontwikkeling. Concepttussendoelen Engels onderbouw vo (http://www.slo.nl/downloads/documenten/ tussendoelen-engels-havo-vwo-onderbouw-vo.pdf/ y http://www.slo.nl/ downloads/documenten/tussendoelen-engels-vmbo-onderbouw-vo.pdf/), consultados el 20 de febrero de 2017.

THIJS, A., TRIMBOS, B., TUIN, D., BODDE, M. en DE GRAAFF, R. (2011): Engels in het basisonderwijs (Enschede, SLO).

TRIMBOS, B. (2007): Concretisering van de kerndoelen Engels. Kerndoelen voor de onderbouw VO (Enschede, SLO).

UNIÓN EUROPEA (2009): Conclusiones del Consejo de 12 de mayo de 2009 sobre un marco estratégico para la cooperación europea en el ámbito de la educación y la formación («ET 2020») (2009/C 119/02). Diario Oficial de la Unión Europea 28.5.2009. 
VAN EK, J. A. (1993): Objectives for foreign language learning (Strasbourg, Council of Europe).

WET VAN 2 JULI 1981, OP HET BASIS ONDERWIJS

WET VAN 29 JANUARI 1993, OP HET VOORTGEZET ONDERWIJS 